\title{
MEGAFAUNA DO PLEISTOCENO SUPERIOR DE SERGIPE, BRASIL: REGISTROS TAXONÔMICOS E CRONOLÓGICOS
}

\author{
MÁRIO ANDRÉ TRINDADE DANTAS \\ Programa de Pós-Graduação em Ecologia e Conservação, UFS, 49100-000, São Cristovão, SE, Brasil. \\ matdantas@yahoo.com.br \\ KLEBERSON DE OLIVEIRA PORPINO \\ Departamento de Ciências Biológicas, Faculdade de Ciências Exatas e Naturais, UERN, Campus Central, Mossoró, RN, \\ Brasil.kleporpino@yahoo.com.br \\ SORAIA GIRARDI BAUERMANN \\ Laboratório de Palinologia, ULBRA, Av. Farroupilha, 8001, 92425-9000, Canoas, RS, Brasil. soraia.bauermann@ulbra.br \\ ANA PAULA DO NASCIMENTO PRATA \\ Laboratório de Sistemática Vegetal, Departamento de Biologia, UFS, UFS, 49100-000, São Cristovão, SE, Brasil. \\ apprata@ufs.br \\ MARIO ALBERTO COZZUOL \\ Laboratório de Paleozoologia, Departamento de Biologia Geral, UFMG, Av. Antonio Carlos, 6627, 31270-010, Belo Horizonte, \\ MG, Brasil.cozzuol@icb.ufmg.br \\ ANGELA KINOSHITA \\ Universidade Sagrado Coração, Rua Irmã Arminda 10-50,17011-160, Bauru, SP, Brasil, angela.kinoshita@ usc.br
}

\begin{abstract}
JEAM HAROLDO OLIVEIRA BARBOSA \& OSWALDO BAFFA
Departamento de Física, Faculdade de Filosofia Ciências e Letras, USP, Av. Bandeirantes 3000, 14040-901, Ribeirão Preto, SP, Brasil.jeamharoldo@hotmail.com,baffa@usp.br
\end{abstract}

\begin{abstract}
LATE PLEISTOCENE MEGAFAUNA FROM SERGIPE, BRAZIL: TAXONOMIC AND CHRONOLOGICAL RECORDS. The Sergipe State is included in the Intertropical Region of Brazil (= IRB), and its fauna of large (biomass $>44 \mathrm{~kg}$ ) and megamammals (biomass $>1000 \mathrm{~kg}$ ) is still unknown. This paper reports three taxa for Fazenda Charco locality: Toxodon platensis Owen, Smilodon populator Lund, and Pachyarmatherium brasiliense Porpino, Fernicola \& Bergqvist; the latter constitutes the first record for this northeastern state of Brazil. Ages for the three faunal localities (Fazenda Elefante, Gararu Municipality; Fazenda Charco and Fazenda São José, Poço Redondo Municipality) are estimated between 50,000 and 38,000 years BP, and this period of time includes two different climates. Finally, the present authors are engaged in an ongoing project to elucidate in more detail the ecology of the species found in the IRB.
\end{abstract}

Key words: Smilodon populator, Pachyarmatherium brasiliense, Toxodon platensis, ESR dating, Quaternary.

\section{INTRODUÇÃO}

Estudos sobre a fauna de mamíferos de grande porte (biomassa $>44 \mathrm{~kg}$ ) e gigantes (biomassa $>1000 \mathrm{~kg}$ ), usualmente chamados de megafauna, que viveram na Região
Intertropical Brasileira - RIB (sensu Cartelle, 1999) ocorrem desde o século XIX. Estes estudos incluem, na sua grande maioria, levantamentos de dados sobre a ocorrência e distribuição desta fauna (e.g. Porpino et al., 2004), além de dados sobre tafonomia dos depósitos fossilíferos (e.g. Santos et al., 2002; Alves et al., 2007), paleopatologia (Melo et al., 2007) e, nos últimos anos, a cronologia desta fauna, através da utilização de métodos alternativos de datação de fósseis ou sedimentos associados (e.g. Faure et al., 1999; Kinoshita et al., 2005, 2008).

Somente nos últimos dez anos pesquisas com esta temática começaram a ocorrer com maior frequência no Estado de Sergipe, focando principalmente no registro de novos táxons e na identificação de faunas locais de tanques e cavernas (e.g. Dantas \& Zucon, 2007; Dantas, 2009). Mesmo assim, nota-se que a fauna de Sergipe ainda é pouco conhecida quando comparada com os demais estados da RIB, como por exemplo, Bahia (Cartelle, 1992), Pernambuco (Oliveira et al., 2009) e Piauí (Guérin et al., 1996).

Os principais objetivos do presente trabalho são: (i) reportar as ocorrências de Toxodon platensis Owen, 1837, Smilodon populator Lund, 1842 e Pachyarmatherium 
brasiliense Porpino, Fernicola \& Bergqvist, 2009 para a localidade Fazenda Charco, Município de Poço Redondo, Estado de Sergipe; (ii) apresentar dados cronológicos inéditos, obtidos através da técnica de Ressonância de Spin Eletrônico (Electron Spin Resonance/ESR), bem como discutir as implicações destas datações no contexto da geocronologia do Pleistoceno da RIB.

\section{MATERIALE MÉTODOS}

O material foi coletado em janeiro de 2009, em um tanque (0946' 58,3"S, 37 40'63,4"O) localizado na Fazenda Charco, Município de Poço Redondo, Sergipe (Figura 1), e atualmente faz parte da coleção científica do Laboratório de Paleontologia da Universidade Federal de Sergipe (LPUFS).

Cinco amostras de fragmentos de dentes pertencentes às espécies Notiomastodon platensis (Ameghino, 1888) [= Stegomastodon waringi (Holand, 1920); Mothé et al., no prelo], Toxodon platensis e Palaeolama major Liais, 1872, tombados na coleção do LPUFS, foram utilizadas para datação pelo método da Ressonância de Spin Eletrônico (ESR) (Tabela 1). Estas amostras são provenientes de três localidades fossilíferas de Sergipe (Fazenda Elefante, Município de Gararu; Fazenda Charco e Fazenda São José, Município de Poço Redondo).

Inicialmente as amostras foram lavadas em água corrente e, posteriormente, submetidas a um banho ultrassônico em água para limpeza superficial. A separação entre esmalte e dentina foi realizada por tratamento térmico. As amostras foram imersas em nitrogênio líquido, até o congelamento completo e, posteriormente, deixadas em temperatura ambiente para o descongelamento. Devido à diferença entre os coeficientes de expansão térmica desses tecidos, o esmalte destaca-se da dentina. Outro fator favorável a este processo é a expansão da água que pode existir entre as camadas. Em algumas amostras esse procedimento teve que ser repetido para a retirada do esmalte e, em outras, a dentina remanescente foi retirada com broca diamantada, acionada por motor de baixa rotação com abundante irrigação em água para evitar o aquecimento. A seguir, as amostras de esmalte foram submetidas a um tratamento químico com ácido clorídrico

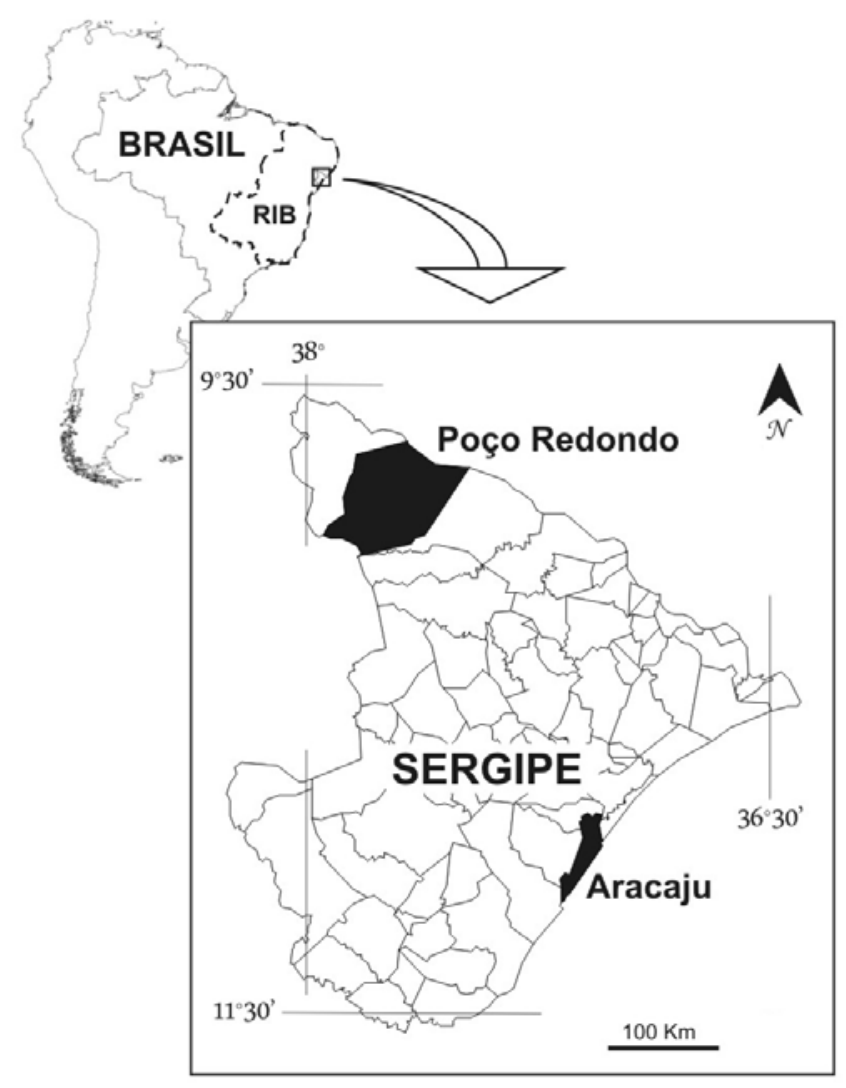

Figura 1. Localização do Município de Poço Redondo, Estado de Sergipe, Brasil. Abreviatura: RIB, Região Intertropical Brasileira.

Figure 1. Location of the Poço Redondo Municipality, Sergipe State, Brazil. Abbreviation: RIB, Intertropical Region of Brazil.

Tabela 1. Amostras de dentes utilizadas para datação por ESR. Dose Equivalente $\left(D_{\mathrm{e}}\right)$ estimada pela curva dose resposta de Toxodon sp. de acordo com Kerber et al. (2011) e resultados das idades. Todas as localidades são do Estado de Sergipe.

Table 1. Teeth samples used for dating by ESR. Equivalent dose $\left(D_{e}\right)$ estimated by the dose-response curve of Toxodon sp. according to Kerber et al. (2011) and outcomes of the ages. All localities are from the Sergipe State.

\begin{tabular}{|c|c|c|c|c|}
\hline $\begin{array}{l}\text { Amostra } \\
\text { dentária }\end{array}$ & Espécie & $\begin{array}{c}\text { Localização } \\
\text { (F. = Fazenda) }\end{array}$ & $\mathrm{D}_{\mathrm{e}}(\mathrm{Gy})$ & $\begin{array}{c}\text { Idade estimada } \\
(\mathrm{ka})\end{array}$ \\
\hline 2 & $\begin{array}{c}\text { Notiomastodon } \\
\text { platensis }\end{array}$ & F. Elefante, Garanu & 50 & 50 \\
\hline 3 & Toxodon platensis & F. Elefante, Gar anu & 50 & 50 \\
\hline 5 & Toxodon platensis & $\begin{array}{c}\text { F. Charco, Poço } \\
\text { Redondo }\end{array}$ & 50 & 50 \\
\hline 6 & Palaeolama major & $\begin{array}{c}\text { F. Charco, Poço } \\
\text { Redondo }\end{array}$ & 38 & 38 \\
\hline 10 & $\begin{array}{l}\text { Notiomastodon } \\
\text { platensis }\end{array}$ & $\begin{array}{c}\text { F. São José, Poço } \\
\text { Redondo }\end{array}$ & 42 & 42 \\
\hline
\end{tabular}


(HCl) na concentração 1:10, por 30 segundos, para limpeza superficial a fim de eliminar contaminação. Nesse processo, uma camada de $250 \mu \mathrm{m}$ é eliminada de cada face do esmalte. Após enxágue em água corrente, as amostras de esmalte foram secas em estufa a $40^{\circ} \mathrm{C}$ e pulverizadas manualmente com gral de ágata e pistilo, onde as partículas adquiriram diâmetro de cerca de $200 \mu \mathrm{m}$.

Os espectros dos esmaltes foram registrados no espectrômetro JEOL FA-200, que opera em Banda X. Cerca de $100 \mathrm{mg}$ foram utilizados para os espectros. Os parâmetros principais de aquisição estão na Tabela 2. Foi utilizado o padrão secundário de Mn para calibrar o campo magnético.

Tabela 2. Parâmetros de aquisição dos espectros.

Table 2. Acquisition parameters of spectra.

\begin{tabular}{cc}
\hline Parâmetros & Valores \\
\hline Campo Central & $338.9 \mathrm{mT}$ \\
Potência de microondas & $2 \mathrm{~mW}$ \\
Varredura & $15 \mathrm{mT}$ \\
Tempo de Varredura & 1 minuto \\
Amplitude de modulação & $0.1 \mathrm{mT}$ \\
Constante de tempo & $100 \mathrm{~ms}$ \\
\hline
\end{tabular}

\section{SISTEMÁTICAPALEONTOLÓGICA}

\author{
NOTOUNGULATA Roth, 1903 \\ TOXODONTIA Owen, 1853 \\ TOXONDONTIDAE Owen, 1845 \\ TOXODONTINAE Trouessart, 1898
}

Toxodon platensis Owen, 1837

(Figuras 2A-E; Tabela 3)

Material. LPUFS 4806, $\mathrm{I}^{1}$; LPUFS 4807, $\mathrm{I}^{1}$; LPUFS 4808, $\mathrm{I}^{2}$; LPUFS 4809, $\mathrm{Pm}^{2}$ esquerdo; LPUFS 4810, $\mathrm{Pm}^{3}$ direito; LPUFS 4811, $\mathrm{Pm}^{4}$ esquerdo; LPUFS 4812, $\mathrm{Pm}^{3}$ direito; LPUFS 4813 a 4816, fragmentos de molares superiores; LPUFS 4817, $\mathrm{M}_{2}$ esquerdo; LPUFS 4818, úmero direito; LPUFS 4823, unciforme direito; LPUFS 4842, metacarpal; LPUFS 4819, metacarpal II esquerdo; LPUFS 4820, metacarpal III; LPUFS 4825, falange proximal do membro anterior; LPUFS 4805, tíbia direita ou esquerda?; LPUFS 4826 e 4827, calcâneos esquerdos; LPUFS 4828, astrágalo esquerdo; LPUFS 4829, astrágalo direito; LPUFS 4841, navicular; LPUFS 4824, falange proximal do membro posterior.

Localização geográfica e idade. Localidade Fazenda Charco, Município de Poço Redondo, Estado de Sergipe, Brasil; Pleistoceno superior.

Comentários. São referidas para o Brasil três espécies
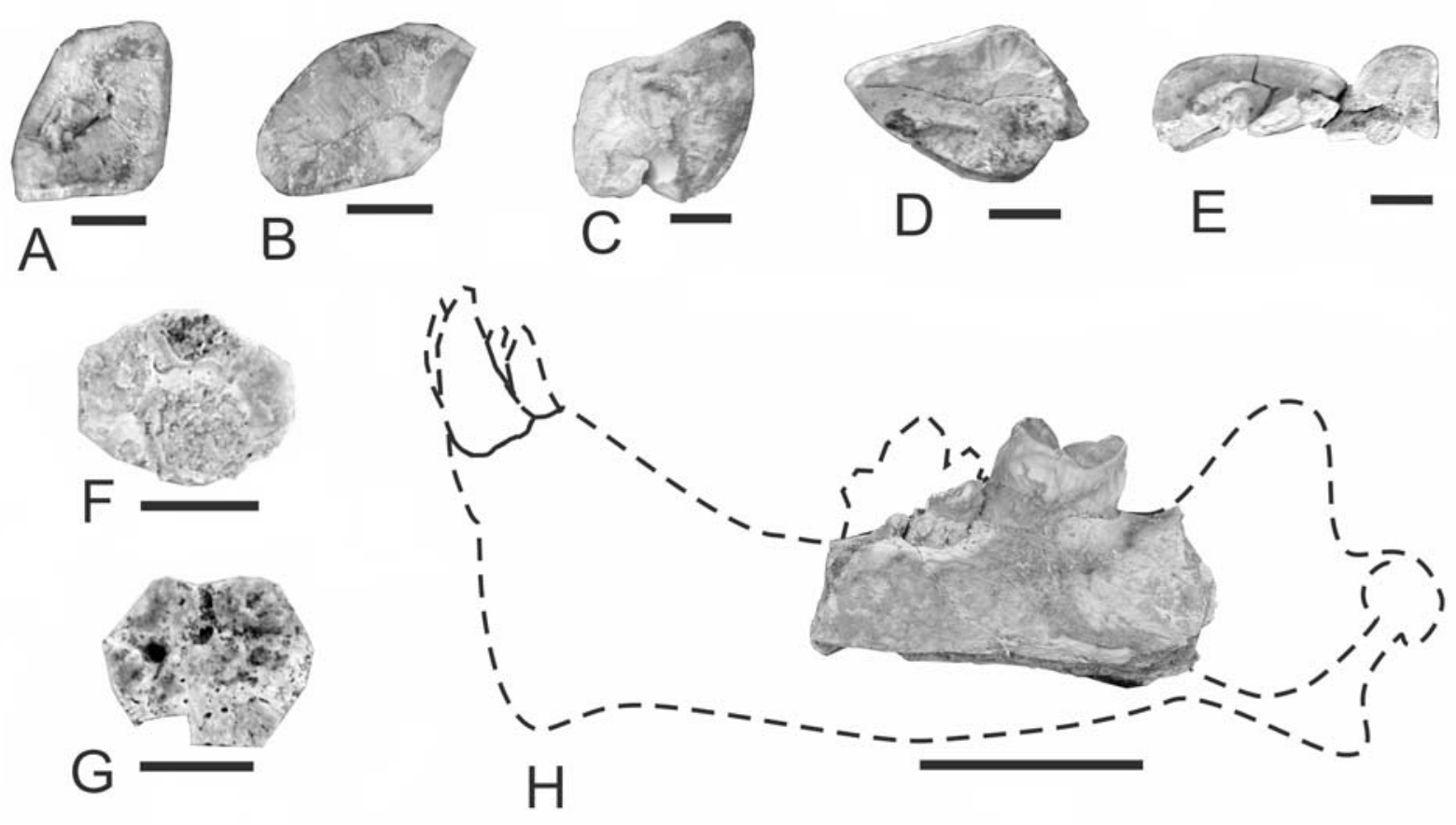

Figura 2. A-E, Toxodon platensis, pré-molares e molares em vista oclusal. A, LPUFS 4809, Pm² esquerdo; B, LPUFS 4812, Pm³ direito; C, LPUFS 4810, $\mathrm{Pm}^{3}$ direito; D, LPUFS 4811, $\mathrm{Pm}^{4}$ esquerdo; E, LPUFS 4817, M 2 esquerdo. F-G, Pachyarmatherium brasiliense. F, LPUFS 4798, osteodermo em vista dorsal; G, LPUFS 4799, osteodermo em vista dorsal. H, Smilodon populator, LPUFS 4832, fragmento de dentário esquerdo em vista lateral. Escalas: $A-G=10 \mathrm{~mm} ; \mathrm{H}=50 \mathrm{~mm}$.

Figure 2. A-E, Toxodon platensis, premolars and molars in occlusal view. A, LPUFS 4809, left Pm²; B, LPUFS 4812, right Pm³; C, LPUFS 4810, right Pm³ ; , LPUFS 4811, left Pm ; E, LPUFS 4817, left $\mathrm{M}_{2}$. F-G, Pachyarmatherium brasiliense. F, LPUFS 4798, osteoderm in dorsal view; G, LPUFS 4799, osteoderm in dorsal view. H, Smilodon populator, LPUFS 4832, left mandibular fragment in lateral view. Scale bars: $A-G=10 \mathrm{~mm} ; \mathrm{H}=50 \mathrm{~mm}$. 
pleistocênicas de toxodontíneos: Toxodon platensis, com ocorrência em quase todo o território brasileiro; Trigodonops lopesi (Roxo, 1921) com registros na Bahia, Minas Gerais e Rondônia (Cartelle, 1992, 1994; Nascimento, 2008); e Mixotoxodon larensis van Frank, 1957, com registro no Acre (Rancy, 1981; Paula-Couto, 1982). Porém, de acordo com Cartelle (1992) Trigodonops engloba duas espécies, T. lopesi e $T$. larensis, esta última representando uma nova combinação para Mixotoxodon larensis. Por outro lado, Mendonça (2007) incluiu em Toxodon duas espécies, T. platensis e T. lopesi.

Tradicionalmente Toxodon e Trigodonops são classificados em subfamílias separadas, Toxodontinae e Haplodontheriinae, respectivamente (Paula Couto, 1979; McKenna \& Bell, 1997). No entanto, Cartelle (1992) classificou ambos os gêneros na subfamília Toxodontinae, o que foi confirmado por análise cladística (Nasif et al., 2000).

Em um estudo prévio, Góes et al. (2002) registraram a ocorrência de Toxodontidae indeterminado na Fazenda Charco. O material aqui descrito, da mesma localidade, é atribuído a Toxodon platensis com base nas características dentárias, onde o $\mathrm{Pm}^{3}$ e $\mathrm{Pm}^{4}$ são bilobulados lingualmente e com uma faixa de esmalte no sulco interlobular, as quais permitem uma diagnose mais clara para as espécies de toxodontíneos, visto que o material pós-craniano aparentemente não apresenta caracteres diagnósticos suficientes para identificação específica (Cartelle, 1992; Miño-Boilini et al., 2006; Mendonça, 2007).

Tabela 3. Comparação das medidas $(\mathrm{mm})$ dos dentes de Toxodon platensis encontrados na Fazenda Charco com os apresentados por (A) Cartelle (1992) e (B) Vidal (1959). Abreviações: 1, diâmetro mesiodistal; 2, diâmetro linguovestibular.

Table 3. Measurements $(\mathrm{mm})$ of teeth of Toxodon platensis found in Fazenda Charco compared with the data from (A) Cartelle (1992) and (B) Vidal (1959). Abbreviations: 1, mesiodistal diameter; 2, linguovestibular diameter.

\begin{tabular}{ccc}
\hline Espécime & 1 & 2 \\
\hline LPUFS 4809 & 19,30 & 19,00 \\
(A) & 23,00 & 21,00 \\
(B) & 36,00 & 27,00 \\
LPUFS 4810 & 24,40 & 24,00 \\
LPUFS 4812 & - & 29,00 \\
(A) & 32,00 & 22,00 \\
LPUFS 4811 & 33,60 & 21,60 \\
(A) & 39,00 & 26,00 \\
(B) & 48,50 & 29,00 \\
LPUFS 4817 & 43,00 & 15,50 \\
(A) & 43,00 & 19,00 \\
(B) & 45,00 & 18,00 \\
\hline
\end{tabular}

CINGULATA Illiger, 1811

Pachyarmatherium Downing \& White, 1995

Pachyarmatherium brasiliense Porpino, Fernicola \& Bergqvist, 2009

(Figuras 2F-G; Tabela 4)

Material. LPUFS 4798 e LPUFS 4799, osteodermos isolados. Localização geográfica e idade. Localidade Fazenda Charco, Município de Poço Redondo, Estado de Sergipe, Brasil; Pleistoceno superior.

Comentários. O gênero Pachyarmatherium foi proposto por Downing \& White (1995) baseado em diversos osteodermos encontrados em sedimentos do Plioceno superior-Pleistoceno inferior da Flórida, EUA. Outros registros do gênero são para o Plioceno final-Pleistoceno inicial da América do Norte e Central (Downing \& White, 1995; Mora et al., 2005), em sedimentos do Pleistoceno superior da Venezuela (Rincón \& White, 2007), e no Brasil, onde há registros nos estados do Rio Grande do Norte (Porpino et al., 2009), Pernambuco (Oliveira et al., 2009) e Rio Grande do Sul (A.M. Ribeiro, com. pes.) também para o Pleistoceno final.

De acordo com Porpino et al. (2009) a posição sistemática deste gênero dentre os Cingulata não é bem definida. Análises cladísticas realizadas por estes autores demonstram que Pachyarmatherium não seria um gliptodonte.

Atualmente são três as espécies reconhecidas neste gênero: Pachyarmatherium leiseyi Downing \& White, 1995, do Plioceno superior-Pleistoceno inferior da América do Norte e Central, P. tenebris Rincón \& White, 2007 e P. brasiliense, do Pleistoceno superior da Venezuela e Nordeste do Brasil, respectivamente (Rincón \& White, 2007; Porpino et al., 2009). Com base nos osteodermos estas últimas espécies diferenciam-se da primeira principalmente pelo tamanho e por figuras periféricas e forames pilíferos mais numerosos.

O material encontrado na Fazenda Charco possui morfologia e medidas concordantes com aquelas de Pachyarmatherium tenebris e P. brasiliense (Tabela 4).

Tabela 4. Comparação das medidas $(\mathrm{mm})$ dos osteodermos de Pachyarmatherium brasiliense com os apresentados por (A) Downing \& White (1995), (B) Rincón \& White (2007) e Porpino et al. (2009). Abreviações: 1, largura; 2, comprimento anteroposterior; 3, espessura.

Table 4. Measurements $(\mathrm{mm})$ of Pachyarmatherium brasiliense osteoderms compared with the data from (A) Downing \& White (1995), (B) Rincón \& White (2007) and Porpino et al. (2009). Abbreviations: 1, lenght; 2, width; 3, thickness.

\begin{tabular}{rccc}
\hline Espécime & 1 & 2 & 3 \\
\hline LPUFS 4798 & 19,0 & 16,0 & 7,0 \\
LPUFS 4799 & 18,0 & 14,5 & 12,5 \\
(A) & $8,1-22,9$ & $8,1-22,9$ & $5,5-13,3$ \\
(B) & $13,0-32,1$ & $14,6-28,5$ & $6,6-11,4$ \\
(C) & $10,1-26,7$ & $14,5-28,0$ & $7,0-18,4$
\end{tabular}


Apesar das semelhanças serem sugestivas de sinonímia para estas espécies, o que daria a prioridade a $P$. tenebris, uma revisão estaria além do escopo do presente trabalho. Por isto, optou-se por atribuir o material aqui estudado a $P$. brasiliense por ser este o táxon descrito para a RIB.

\section{CARNIVORA Bowdich, 1821 FELIDAE Fischer, 1817 MACHAIRODONTINAE Gill, 1872}

\section{Smilodon populator Lund, 1842}

(Figuras 2H; Tabelas 5, 6)

Material. LPUFS 4832, fragmento de dentário esquerdo; LPUFS 4833, rádio esquerdo; LPUFS 4834, metacarpal II (?). Localização geográfica e idade. Localidade Fazenda Charco, Município de Poço Redondo, Estado de Sergipe, Brasil; Pleistoceno superior.

Comentários. A classificação dos tigres-dente-de-sabre da América é discutível, entretanto são aqui reconhecidas quatro

Tabela 5. Comparação das medidas $(\mathrm{mm})$ do $\mathrm{M}_{1}$ de Smilodon populator encontrado na Fazenda Charco com os apresentados por (A) Paula Couto (1955). Abreviações: 1, diâmetro mesiodistal; 2, diâmetro linguovestibular.

Table 5. $\mathrm{M}_{1}$ measurements $(\mathrm{mm})$ of Smilodon populator found in Fazenda Charco compared with the data from (A) Paula Couto (1955). Abbreviations: 1, mesiodistal diameter; 2, linguovestibular diameter.

\begin{tabular}{ccc}
\hline Espé cime & 1 & 2 \\
\hline LPUFS 4832 & 28,3 & 12,7 \\
(A) & 30,0 & 14,0 \\
\hline
\end{tabular}

Tabela 6. Comparação das medidas $(\mathrm{mm})$ do rádio e do metacarpo de Smilodon populator com os apresentados por (A) Merriam \& Stock (1932) e (B) Castro \& Langer (2008). Abreviações: 1, comprimento ântero-posterior; 2, diâmetro transversal da extremidade proximal; 3, diâmetro ântero-posterior da extremidade proximal; 4, diâmetro transversal da extremidade distal; $\mathbf{5}$, diâmetro antero-posterior da extremidade distal; (1) média das medidas máxima e mínimas apresentadas; ${ }^{(2)}$ medidas incompletas devido a fragmentação da peça.

Table 6. Measurements $(\mathrm{mm})$ of radius and metacarpal of Smilodon populator compared with the data from (A) Merriam \& Stock (1932) and (B) Castro \& Langer (2008). Abbreviations: 1, anteroposterior lenght; 2, transversal diameter of the proximal end; $\mathbf{3}$, anteroposterior diameter of the proximal end; $\mathbf{4}$, transversal diameter of the distal end; 5 , anteroposterior diameter of the distal end; (1) average of maximum and minimum measurements; ${ }^{(2)}$ measurement compromised by fracture.

\begin{tabular}{cccccc}
\hline Espécime & 1 & 2 & 3 & 4 & 5 \\
\hline LPUFS 4833 & 268,0 & 43,0 & 31,1 & 59,0 & 34,6 \\
$(\mathrm{~A})^{(1)}$ & 265,0 & 48,4 & 38,1 & 58,3 & 41,9 \\
$(\mathrm{~B})$ & 270,0 & - & - & 75,0 & 50,0 \\
LPUFS 4834 & $84,2^{(2)}$ & - & - & 20,4 & 18,2 \\
(A) & 90,9 & 21,3 & 30,6 & 24,8 & - \\
\hline
\end{tabular}

espécies pertencentes aos gêneros Smilodon ( $S$. gracilis Cope, 1888, S. fatalis Leidy, 1868, S. populator Lund, 1842) e Homotherium (H. venezuelensis Rincón, Prevosti \& Parra, 2011). S. fatalis e S. populator são registradas em toda a América; $S$. gracilis, na América do Norte e norte da América do Sul (Venezuela), e H. venezuelensis apenas na América do Sul (Venezuela e Uruguai?) (Prevosti \& Pomi, 2007; Castro \& Langer, 2008; Rincón et al., 2011).

No Brasil, até o momento, é registrada apenas a espécie Smilodon populator (Prevosti \& Pomi, 2007; Castro \& Langer, 2008), sendo que na região Nordeste é ocorrente em várias localidades pleistocênicas, em todos os estados, com exceção do Maranhão. Em Sergipe foi registrada por Dantas et al. (2005) para o Município de Gararu. Portanto, o material aqui estudado apesar da sua escassez e de não apresentar características morfológicas significativas, é atribuído tentativamente a $S$. populator, levando em consideração o seu tamanho, equivalente aos dos registros anteriores desta espécie no Brasil.

\section{CRONOLOGIA E INTERPRETAÇÃO PALEOAMBIENTAL}

A quantidade de esmalte, obtida dos dentes de mamíferos das localidades Fazenda Elefante, Fazenda Charco e Fazenda São José, foi pequena e em virtude disto não foi possível construir uma curva de calibração para as amostras analisadas. Portanto, a primeira estimativa aproximada das idades dos dentes estudados foi realizada através da curva dose-resposta de material semelhante, atribuído a Toxodon sp. encontrado na Formação Touro Passo, Município de Uruguaiana, Rio Grande do Sul (Kerber et al., 2011). Pressupõe-se que o esmalte dos fósseis possui a mesma sensibilidade à radiação ionizante. Essa hipótese já foi empregada em experimentos para se determinar dose de dentes expostos à radiação depositada em humanos, e também em esmalte de dentes de mamíferos fósseis, mostrando-se válida.

A partir dos valores das intensidades da amplitude pico a pico $\left(\mathrm{A}_{\mathrm{pp}}\right)$, obtidos através dos espectros (vide Figura $3)$, pôde-se estimar a Dose Equivalente $\left(D_{e}\right)$ de cada amostra, através da curva dose-resposta em Toxodon sp. (Kerber et al., 2011). Os resultados encontram-se na Tabela 1. Para a conversão de $\mathrm{D}_{\mathrm{e}}$ em idade, foram necessários os valores das taxas de dose externa, devido à radiação cósmica e radioisótopos presentes no sedimento e na dentina, e a taxa de dose interna, devido aos radioisótopos presentes no esmalte. Na conversão de $D_{\text {e }}$ para idade, utilizou-se a taxa de dose de $1 \mathrm{mGy} / \mathrm{ano}$, que foi o valor encontrado para a amostra de Toxodon sp., da Formação Touro Passo, Rio Grande do Sul. Este valor corresponde a taxas de doses típicas que foram encontradas em outros trabalhos de datação (Kinoshita et al., 2002, 2005, 2008; Baffa et al., 2006), sendo considerada uma aproximação razoável (ver Tabela 1). 


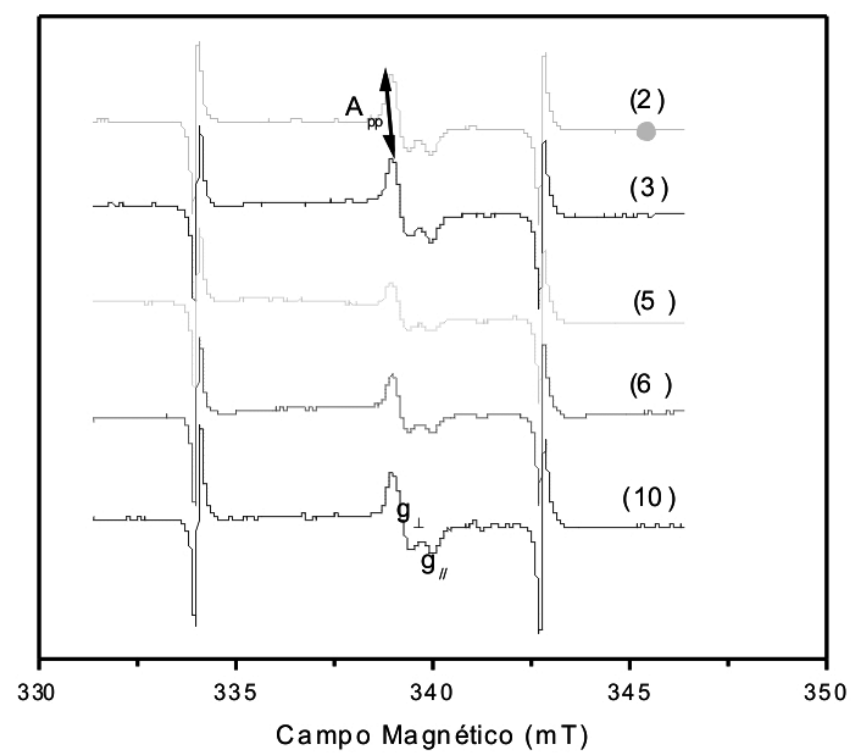

Figura 3. Espectro ESR do esmalte dos dentes 2, 3, 5, 6 e 10. Observa-se o espectro do radical gerado pela radiação ionizante $\left(\mathrm{CO}_{2}{ }^{-}\right)$com fatores espectroscópicos $\mathrm{g}_{\Lambda}=2.0018$ e $\mathrm{g}_{/ /}=1.9973$ entre a terceira e quarta linhas do íon Manganês $\left(\mathrm{Mn}^{2+}\right)$. A amplitude pico a pico $\left(A_{p p}\right)$ foi utilizada como parâmetro para avaliar a idade do fóssil.

Figure 3. ESR spectrum of tooth enamel 2, 3, 5, 6, 10. Observe the spectrum generated by ionizing radiation $\left(\mathrm{CO}_{2}^{-}\right)$with spectroscopic factors $g_{\Lambda}=2.0018$ e $g_{\mu}=1.9973$ between the third and fourth lines of manganese ion $\left(\mathrm{Mn}^{2+}\right)$. The peak amplitude $\left(\mathrm{A}_{\mathrm{pp}}\right)$ was used as parameter for assessing the age of the fossil.

As idades estimadas encontradas nos fósseis coletados em tanques do Estado de Sergipe (Tabela 1) situam estes depósitos em um período temporal entre 50.000 e 38.000 anos AP, encontrando uma idade próxima as datações realizadas em tanques de outras localidades da RIB, como em Alagoas (Silva, 2009), Paraíba (Kinoshita et al., 2005) e Pernambuco (Kinoshita et al., 2008), como pode ser observado na Tabela 7.

Estes dados, em conjunto, mostram que os tanques preservaram restos faunísticos de um intervalo temporal entre 60 e 10 mil anos, enquanto cavernas apresentam um intervalo temporal mais amplo, entre 350 e 10 mil anos AP (Tabela 7) (Auler et al., 2006).

Estas datações, ainda que pontuais, permitem considerar as idades das assembleias fossilíferas dos depósitos estudados no contexto da escala cronológica da América do Sul fornecida pela área pampeana da Província de Buenos Aires, Argentina, em que são reconhecidas quatro idades para o Pleistoceno: Ensenadense, Bonaerense, Lujanense e Platense (Cione \& Tonni, 1999, 2005).

Os tanques apresentariam fósseis de idade equivalente aos do Lujanense, enquanto as cavernas possuiriam restos faunísticos de idades equivalentes aos do Bonaerense, Lujanense e Platense. A atribuição da idade Lujanense à fauna encontrada em tanques e cavernas foi tentada anteriormente através do estabelecimento de faunas-locais (e.g. Bergqvist
Tabela 7. Cronologia da fauna de mamíferos (encontrados na Fazenda Charco, Poço Redondo) registrados na Região Intertropical Brasileira. Abreviações: A, Eremotherium laurillardi; B, Catonyx cuvieri; C, Notiomastodon platensis; D, Toxodon platensis; E, Palaeolama major, F, Smilodon populator, (1) Auler et al. (2006); (2) Neves \& Piló (2003); (3) Kinoshita et al. (2008); (4) dados do presente trabalho; (5) Silva (2009); (6) Kinoshita et al. (2005); (*) Idade anterior de 350.000 anos AP realizado por Auler et al. (2006).

Table 7. Chronology of mammals (found in Fazenda Charco, Poço Redondo) reported for the Brazilian intertropical region. Abbreviations: A, Eremotherium laurillardi; B, Catonyx cuvieri; C, Notiomastodon platensis; D, Toxodon platensis; E, Palaeolama major, F, Smilodon populator, ${ }^{(1)}$ Auler et al. (2006); ${ }^{(2)}$ Neves \& Piló (2003); ${ }^{(3)}$ Kinoshita et al. (2008); (4) data from the present work; ${ }^{(5)}$ Silva (2009); (6) Kinoshita et al. (2005);. (*) previous age of 350,000 years BP conducted by Auler et al. (2006).

\begin{tabular}{|c|c|c|c|c|c|c|}
\hline \multirow{2}{*}{ Idades } & \multicolumn{6}{|c|}{ Táxons } \\
\hline & A & $\mathrm{B}$ & $\mathrm{C}$ & $\mathrm{D}$ & E & $\mathrm{F}$ \\
\hline 10.000 & (1) & (2) & (5) & & & (2) \\
\hline 15.000 & & & & & & \\
\hline 20.000 & & & & & & \\
\hline 25.000 & & (1) & (6) & & & \\
\hline 30.000 & & & & & & \\
\hline 35.000 & & & & & & \\
\hline 40.000 & & & & (4) & (4) & \\
\hline 45.000 & & & (5) & & & \\
\hline 50.000 & & & (4) & & & \\
\hline 55.000 & & & & & & \\
\hline 60.000 & & & (3) & & & \\
\hline 65.000 & & & & & & \\
\hline 70.000 & & & & & & \\
\hline 75.000 & & & & & & \\
\hline 80.000 & & & & & & \\
\hline 85.000 & & & & & & \\
\hline 90.000 & & & & & & \\
\hline 95.000 & & & & & & \\
\hline 100.000 & & & & & & \\
\hline 105.000 & & & & & & \\
\hline 110.000 & & & & & & \\
\hline 115.000 & & & & & & \\
\hline 120.000 & & & & & & \\
\hline 125.000 & & (1) & & & $\left(^{*}\right)$ & \\
\hline
\end{tabular}


et al., 1997), entretanto, a grande distância geográfica entre a RIB e a Argentina, e a presença de elementos distintos entre estas duas faunas, como por exemplo, Eremotherium laurillardi (Lund, 1842), Catonyx cuvieri (Lund, 1839) e Pachyarmatherium brasiliense, torna esta inferência pouco informativa, tendo a utilização destas idades sido desaconselhada por alguns autores (e.g. Cartelle, 1999).

Com base na presença dos táxons acima referidos na RIB, em um período cronológico mais amplo do que o suposto anteriormente (e.g. Cartelle, 1999), é possível discutir quanto ao tipo de ambientes a que estavam associados, dentro do contexto das mudanças climáticas que ocorreram durante o Pleistoceno. A megafauna encontrada em Sergipe apresenta 11 táxons de mamíferos de grande porte (biomassa $>44 \mathrm{~kg}$ ) e gigantes (biomassa $>1000 \mathrm{~kg}$ ) que viveram nesta região durante o Pleistoceno superior (Tabela 8). Esta associação faunística se repete em outros estados no âmbito da RIB (e.g. Pernambuco, Alagoas e Bahia) e geralmente é considerada indicadora de áreas abertas. Por outro lado, conforme Oliveira et al. (2009) o clima é mais quente e úmido para a fauna do tanque de Fazenda Nova, PE, e isto indicaria área mais florestada.

Dados paleoclimáticos para a RIB (Behling et al., 2000;
Barreto, 2010) demonstram que as áreas abertas não estiveram intactas durante todo o Pleistoceno, havendo movimentos de expansão e retração (Cione et al., 2003; 2007). As evidências isotópicas e palinológicas existentes (Behling et al., 2000; Barreto, 2010) sobre a RIB indicam que durante o Pleistoceno superior houve, pelo menos, três episódios de mudanças ambientais em que ocorreram intercalação de condições climáticas distintas.

De acordo com Barreto (2010) em um período compreendido entre 90.000 e 40.000 anos AP houve o predomínio de clima úmido, onde as áreas abertas sofreram retração com a expansão das florestas pluviais. Posteriormente, há aproximadamente 40.000 anos AP, iniciou uma nova mudança climática, com predomínio de clima quente e seco (Barreto, 2010), ocorrendo novamente expansão de áreas abertas, e na qual a vegetação predominante deveria ser a de Caatinga (De Oliveira et al., 2005). Os dados cronológicos disponíveis (Tabela 7) sugerem que a megafauna viveu durante ambos os episódios sob regimes climáticos e condições ambientais distintos. É interessante observar que estudos isotópicos de esmalte dentário de Toxodon sugerem que este megaherbívoro, frequente em assembleias do Pleistoceno da RIB e classicamente

Tabela 8. Táxons de grandes mamíferos (biomassa $>44 \mathrm{~kg}$ ) e mega mamíferos (biomassa $>1000 \mathrm{~kg}$ ) ocorrentes no Estado de Sergipe.

Table 8. Taxa of large mammals (body mass $>44 \mathrm{~kg}$ ) and megamammals (body mass $>1000 \mathrm{~kg}$ ) with occurrence in the Sergipe State.

\begin{tabular}{ll}
\hline Grandes mamíferos & Mega mamíferos \\
\hline XENARTHRA & XENARTHRA \\
Mylodontidae & Megatheriidae \\
Mylodontinae indeterminado & Eremotherium laurillardi \\
Catonyx cuvieri & Glyptodontidae \\
CINGULATA & Glyptodon clavipes \\
Pachyarmatherium brasiliense & Glyptotherium sp. \\
CARNIVORA & PROBOSCIDEA \\
Felidae & Gomphotheriidae \\
Smilodon populator & Notiomastodon platensis \\
PERISSODACTYLA & NOTOUNGULATA \\
Equidae & Toxodontidae \\
Equinae indeterminado & Toxodon platensis \\
ARTIODACTYLA & \\
\hline Camelidae & \\
\hline
\end{tabular}


interpretado como pastador seria na verdade ecologicamente mais versátil e capaz de subsistir em ambientes variando de florestas tropicais a savanas (MacFadden, 2005). Deste modo, as inferências paleoambientais tradicionalmente feitas com base apenas na presença de elementos da megafauna em tanques e cavernas da RIB devem ser consideradas com cautela.

\section{CONSIDERAÇÕES FINAIS}

Na Fazenda Charco foram encontrados, em associação, fósseis dos táxons Eremotherium laurillardi, Catonyx cuvieri, Notiomastodon platensis, Palaeolama major, Toxodon platensis, Pachyarmatherium brasiliense e Smilodon populator, sendo estes três últimos registrados nas coletas realizadas para o presente trabalho.

As estimativas das idades obtidas através de datação por ESR em mamíferos pleistocênicos de tanques de três localidades no Estado de Sergipe indicam um intervalo temporal entre 50.000 e 38.000 anos AP, similar às idades obtidas em outras áreas na RIB, posicionando a paleofauna encontrada durante dois períodos climáticos distintos, e possivelmente vivendo associada tanto à vegetação de áreas abertas, quanto à vegetação de ambiente florestado.

\section{AGRADECIMENTOS}

A CAPES pela bolsa de estudos, nível mestrado, fornecida ao primeiro autor; ao CNPq (edital MCT/CNPq n ${ }^{\circ} 032 / 2010$ ) e FAPERN (edital 013/2009-PPP III) pelo apoio financeiro fornecido ao segundo autor; e aos revisores anônimos pelas sugestões de correção que permitiram o aprimoramento do manuscrito.

\section{REFERÊNCIAS}

Alves, R. da S.; Barreto, A.M.F.; Borges, L.E.P. \& Farias, C.C. 2007. Aspectos tafonômicos no depósito de mamíferos pleistocênicos de Brejo da Madre de Deus, Pernambuco. Estudos Geológicos, 17(2):114-122.

Auler, A.S.; Piló, L.B.; Smart, P.L.; Wang, X.; Hoffmann, D.; Richards, D.A.; Edwards, R.L.; Neves, W.A. \& Cheng, H. 2006. U-series dating and taphonomy of Quaternary vertebrates from Brazilian caves. Palaeogeography, Palaeoclimatology, Palaeoecology, 240:508-522. doi:10.1016/j.palaeo.2006.03.002

Baffa, O.; Kinoshita, A.; Figueiredo, A.M.G.; Brunetti, A. \& Ginesu, S., 2006. ESR dating of an ancient goat tooth from Nuoro, Sardinia. Italy Radiation Protection Dosimetry, 119(1-4):446449. doi: $10.1093 /$ rpd/nci502

Barreto, E.A. de S. 2010. Reconstituição da pluviosidade da Chapada Diamantina (BA) durante o Quaternário tardio através de registros isotópicos $(\mathrm{O}$ e $\mathrm{C})$ em estalagmites. Programa de Pós-Graduação em Geoquímica e Geotectônica, Universidade de São Paulo, Dissertação de Mestrado, 133 p.

Behling, H.; Arz, H.W.; Pätzold, J.R. \& Wefer, G. 2000. Late Quaternary vegetational and climate dynamics in northeastern
Brazil, inferences from marine core GeoB 3104-1. Quaternary Science Reviews, 19:981-994.

Bergqvist, L.P.; Gomide, M.; Cartelle, C. \& Capilla, R. 1997. Faunas locais e mamíferos pleistocênicos de Itapipoca/Ceará, Taperoa/ Paraíba, e Campina Grande/Paraíba. Estudo comparativo, bioestratinômico e paleoambiental. Revista Universidade Guarulhos, Geociências, 2(6):23-32.

Cartelle, C. 1992. Edentata e megamamíferos herbívoros extintos da toca dos ossos (Ourolândia, BA). Programa de Pós-Graduação em Morfologia, Universidade Federal de Minas Gerais, Tese de Doutorado, $516 \mathrm{p}$.

Cartelle, C. 1994. Tempo passado: mamíferos do Pleistoceno de Minas Gerais. Belo Horizonte, Editora Palco, 131 p.

Cartelle, C. 1999. Pleistocene mammals of the Cerrado and Caatinga of Brazil. In: J.F. Eisenberg \& K.H. Redford (eds.) Mammals of the Neotropics, The University of Chicago Press, p. 27-46.

Castro, M.C. de \& Langer, M.C. 2008. New postcranial remains of Smilodon populator Lund, 1842 from south-central Brazil. Revista Brasileira de Paleontologia, 11(3):199-206. doi:10.4072/rbp.2008.3.06

Cione, A.L. \& Tonni, E.P. 1999. Biostratigraphy and chronological scale of upper-most Cenozoic in the Pampean Area, Argentina. In: J. Rabassa \& M. Salemme (eds.) Quaternary of South America and Antarctic Peninsula, A.A. Balkema Publishers, p. 23-52.

Cione, A.L. \& Tonni, E.P. 2005. Bioestratigrafía basada em mamíferos del Cenozoico Superior de la Província de Buenos Aires, Argentina. In: R.E. Barrio; R.O. Etcheverry; M.F. Caballé \& E. Llambias (eds.) Geología y recursos minerales de la Província de Buenos Aires, Quick Press, p. 183-200.

Cione, A.L.; Tonni, E.P. \& Soibelzon, L. 2003. The broken zig zag: late Cenozoic large mammals and tortoise extinction in South America. Revista del Museo Argentino de Ciencias Naturales, 5(1):1-19.

Cione, A.L.; Tonni, E.P. \& Soibelzon, L. 2007. Did humans cause the late Pleistocene-early Holocene mammalian extinctions in South America in a context of shrinking open areas? In: G. Haynes (ed.) American megafaunal extinctions at the end of the Pleistocene, Springer Science/Business Media, p. 125-144.

Dantas, M.A.T. \& Zucon, M.H. 2007. Occurrence of Catonyx cuvieri (Lund, 1839) (Tardigrada, Scelidotheriinae) in Late Pleistocene-Holocene of Brazil. Revista Brasileira de Paleontologia, 10(2):129-132.

Dantas, M.A.T. 2009. Primeiro registro de fósseis de mamíferos do Pleistoceno final-Holoceno em cavernas do Estado de Sergipe, Brasil. Revista Brasileira de Paleontologia, 12(2):161-164. doi:10.4072/rbp.2009.2.06

Dantas, M.A.T.; Zucon, M.H. \& Ribeiro, A.M. 2005. Megafauna pleistocênica de Gararu, Sergipe, Brasil. Revista Geociências (UNESP), 24(3):277-287.

De-Oliveira, P.E.; Behling, H.; Ledru, M.P.; Barberi, M.; Bush, M. Salgado-Labouriau, M.L.; Garcia, M.J.; Medeanic, S.; Barth, O.M.; Barros, M.A. de. \& Scheel-Ybert, R. 2005. Paleovegetação e paleoclimas do Quaternário do Brasil. In: C.R. de G. Souza; K. Suguio; A.M. dos S. Oliveira \& P.E. De-Oliveira (eds.) Quaternário do Brasil, Ribeirão Preto, Holos Editora, p. 52-74.

Downing, K.F. \& White, R.S. 1995. The cingulates (Xenarthra) of the Leisey Shell Pit local fauna (Irvingtonian), Hillsborough 
County, Florida. Bulletin of the Florida Museum of Natural History, 37:375-396.

Faure, M.; Guérin, C. \& Parenti, F. 1999. Découverte d'une mégafaune holocène à la Toca do Serrote do Artur (aire archéologique de São Raimundo Nonato, Piauí, Brésil). Comptes Rendus de l'Académie des Sciences, 329:443-448.

Goes, F.A.S.; Vieira, F.S.; Zucon, M.H.; Cartelle, C. \& Teodósio, C. 2002. Ocorrência de mamíferos pleistocênicos em Sergipe, Brasil. Arquivos do Museu Nacional, 60(3):199-206.

Guérin, C.; Curvello, M.A.; Faure, M.; Hugueney, M. \& MourerChauviré, C. 1996. A fauna pleistocênica do Piauí (Nordeste do Brasil): relações paleoecológicas e biocronológicas. Fundhamentos, 3:56-103.

Kerber, L.; Kinoshita, A.; José, F.A.; Figueiredo, A.M.G.; Oliveira, E.V. \& Baffa, O. 2011. Electron Spin Resonance dating of the southern Brazilian Pleistocene mammals from Touro Passo Formation, and remarks on the geochronology, fauna and paleoenvironments. Quaternary International, 245(2):201-208. doi:10.1016/j.quaint.2010.10.010

Kinoshita, A.; Barreto, A.; Alves, R.; Figueiredo, A.M.; Sarkis, J.E. de S.; Dias, M.L. \& Baffa, O. 2008. ESR dating of teeth from northeastern Brazilian megafauna. Radiation Measurements, 43:809-812. doi:10.1016/j.radmeas.2007.11.075

Kinoshita, A.; Brunetti, A.; Avelar, W.E.P.; Mantelatto, F.L.M.; Simões, M.G.; Fransozo, A. \& Baffa, O. 2002. ESR dating of a subfossil shell from Couve Island, Ubatuba, Brazil. Applied Radiation and Isotopes, 57(4):497-500. doi:10.1016/S09698043(02)00096-9

Kinoshita, A.; Franca, A.M.; Almeida, J.A.C. de; Figueiredo, A.M.; Nicolucci, P.; Graeff, C.F.O. \& Baffa, O. 2005. ESR dating at K and $\mathrm{X}$ band of northeastern Brazilian megafauna. Applied Radiation and Isotopes, 62:225-229. doi:10.1016/ j.apradiso.2004.08.007

MacFadden, B.J. 2005. Diet and habitat of toxodont megaherbivores (Mammalia, Notoungulata) from the late Quaternary of South and Central America. Quaternary Research, 64:113-124. doi:10.1016/j.yqres.2005.05.003

McKenna, M.C. \& Bell, S.K. 1997. Classification of mammals above the species level. Nova Yorque, Columbia University Press, $631 \mathrm{p}$.

Melo, D.J. de; Henriques, D.D.R. \& Carvalho, C.R. 2007. Ocorrência de defeitos de esmalte em materiais de Toxodon na coleção do Museu Nacional/UFRJ, uma perspectiva paleoepidemiológica. In: I.S. Carvalho; R. de C.T. Cassab; C. Schwanke; M. de A. Carvalho; A.C.S. Fernandes; M.A. da C. Rodrigues; M.S.S. Carvalho; M. Arai \& M.E.Q. Oliveira (orgs.) Paleontologia: Cenários da Vida, Editora Interciência, 1:745-756.

Mendonça, R. 2007. Revisão dos toxodontes pleistocênicos brasileiros e considerações sobre Trigodonops lopesi (Roxo, 1921) (Notoungulata, Toxodontidae). Programa de PósGraduação em Zoologia, Instituto de Biociências da Universidade de São Paulo, Dissertação de Mestrado, 104 p.

Merriam, J.C. \& Stock, C. 1932. The Felidae of Rancho La Brea. Carnegie Institution of Washington, 422:1-231.

Miño Boilini, A.R., Cerdeño, E. \& Bond, M. 2006. Revisión del género Toxodon Owen, 1837 (Notoungulata: Toxodontidae) en el Pleistoceno de las provincias de Corrientes, Chaco y Santa Fe, Argentina. Revista Española de Paleontología, 21(2):93-103.
Mora, C.L.; Zamora, A.L.V. \& Gamboa, E.A.P. 2005. Los xenarthras fósiles de la localidad de Buenos Aires de Palmares (Blancano Tardío-Irvingtoniano Temprano), provincia de Alajuela, Costa Rica. Revista Geológica de América Central, 33:83-90.

Mothé, D.; Avilla, L.S.; Cozzuol, M.A. \& Winck, G.R. no prelo. Revision of the Quaternary South America lowland gomphotheres (Mammalia: Proboscidea: Gomphotheriidae). Quaternary International. doi:10.1016/j.quaint.2011.05.018

Nascimento, E.R. do. 2008. Os Xenarthra Pilosa (Megatheriidae), Notoungulata (Toxodontidae) e Proboscidea (Gomphotheriidae) da Formação Rio Madeira, Pleistoceno Superior, Estado de Rondônia, Brasil. Programa de Pós-Graduação em Geociências, Universidade Federal do Rio Grande do Sul, Dissertação de Mestrado, $113 \mathrm{p}$.

Nasif, N.L.; Musalem, S. \& Cerdeño, E. 2000. A new toxodont from the Late Miocene of Catamarca, Argentina, and a phylogenetic analysis of the Toxodontidae. Journal of Vertebrate Paleontology, 20(3):591-600.

Neves, W.A. \& Piló, L.B. 2003. Solving Lund's dilemma: new AMS dates confirm that humans and megafauna coexisted at Lagoa Santa. Current Research in the Pleistocene, 20:57-60.

Oliveira, E.V.; Barreto, A.M.F. \& Alves, R. da S. 2009. Aspectos sistemáticos, paleobiogeográficos e paleoclimáticos dos mamíferos Quaternários de Fazenda Nova, PE, Nordeste do Brasil. Gaea, 5(2):75-85. doi:10.4013/gaea.2009.52.04

Paula Couto, C. de. 1955. O "Tigre-dentes-de-Sabre" do Brasil. Boletim do Conselho Nacional de Pesquisas, 1:1-30.

Paula Couto, C. de. 1979. Tratado de Paleomastozoologia. Rio de Janeiro, Academia Brasileira de Ciências, 590 p.

Paula Couto, C. de. 1982. Fossil mammals from the Cenozoic of Acre, Brazil. V. Notoungulata Nesodontinae (II) Haplodontheriinae and Liptoterna, Pyrotheria and Astrapotheria (II). Iheringia, Série Geológica, 7:5-43.

Porpino, K. de O.; Fernicola, J.C. \& Bergqvist, L.P. 2009. A new Cingulate (Mammalia: Xenarthra) Pachyarmatherium brasiliense sp. nov. from the late Pleistocene of northeastern Brazil. Journal of Vertebrate Paleontology, 29(3):881-893.

Porpino, K.O.; Santos, M. de F.C.F. dos \& Bergqvist, L.P. 2004. Registros de mamíferos fósseis no Lajedo de Soledade, Apodi, Rio Grande do Norte, Brasil. Revista Brasileira de Paleontologia, 7(3):349-358.

Prevosti, F.J. \& Pomi, L.H. 2007. Revisión sistemática y antigüedad de Smilodontidion riggi (Carnivora, Felidae, Machairondotinae). Revista del Museo Argentino de Ciencias Naturales, 9(1):67-77.

Rancy, A. 1981. Mamíferos fósseis do Cenozóico do Alto Juruá, Acre. Programa de Pós-Graduação em Geociências, Universidade Federal do Rio Grande do Sul, Dissertação de Mestrado, 122 p.

Rincón, A.D. \& White, R. 2007. Los Xenarthra Cingulata del Pleistoceno tardio (Lujanense) de Cerro Misión, estado Falcón, Venezuela. Boletín de la Sociedad Venezolana de Espeleología, 41:2-12.

Rincón, A.D.; Prevostri, F.J. \& Parra, G.E. 2011. New saber-toothed cat records (Felidae: Machairodontinae) for the Pleistocene of Venezuela, and the great American biotic interchange. Journal of Vertebrate Paleontology, 31(2):468-478.

Santos, M. de F.C.F. dos; Bergqvist, L.P.; Lima-Filho, F.P. \& Pereira, M.M.V. 2002. Feições tafonômicas observadas em fósseis Pleistocênicos do Rio Grande do Norte. Revista de Geologia, 15:31-41. 
Silva, J.L.L. de. 2009. Reconstituição paleoambiental baseada no estudo de mamíferos pleistocênicos de Maravilha e Poço das Trincheiras, Alagoas, Nordeste do Brasil. Programa de PósGraduação em Geociências, Universidade Federal de Pernambuco, Tese de Doutorado, 244 p.

Van Frank, R. 1957. A fossil collection from northern Venezuela 1.
Toxodontidae (Mammalia, Notoungulata). American Museum Novitates, 1850:1-38.

Vidal, N. 1959. Um Toxodon no Pleistoceno de Pernambuco. Boletim do Museu Nacional, 30:1-17.

Received in December, 2010; accepted in November, 2011. 